\title{
ARTIFACT FREE SIGNAL DENOISING WITH WAVELETS
}

\author{
Sylvain Durand
}

CMLA,

Ecole Normale Supérieure de Cachan, 61 avenue du Président Wilson, 94235 Cachan cedex, France. sdurand@cmla.ens-cachan.fr
Jacques Froment

\author{
PRISME, UFR Math. et Info. \\ Université Paris 5 R. Descartes \\ 45 rue des Saints-Pères, \\ 75270 Paris cedex 06, France. \\ froment@math-info.univ-paris5.fr
}

\begin{abstract}
Recent years have seen the development of signal denoising algorithms based on wavelet transform. It has been shown that thresholding the wavelet coefficients of a noisy signal allows to restore the smoothness of the original signal. However, wavelet denoising suffers of a main drawback : around discontinuities the reconstructed signal is smoothed, exhibiting pseudo-Gibbs phenomenon. We consider the problem of denoising piecewise smooth signals with sharp discontinuities. We propose to apply a traditional wavelet denoising method and to restore the denoised signal using a total variation minimization approach. This second step allows to remove the Gibbs phenomena and therefore to restore sharp discontinuities, while the other structures are preserved. The main innovation of our algorithm is to constrain the total variation minimization by the knowledge of the remaining wavelet coefficients. In this way, we make sure that the restoration process does not deteriorate the information that has been considered as significant in the denoising step. With this approach we substantially improve the performance of classical wavelet denoising algorithms, both in terms of SNR and in terms of visual artifacts.
\end{abstract}

\section{INTRODUCTION AND BACKGROUND}

Since the beginning of wavelet transforms in signal processing, it has been noticed that wavelet thresholding is of considerable interest for removing noise from signals and images. The method consists to decompose the noisy data into an orthogonal wavelet basis, to suppress the wavelet coefficients smaller than a given amplitude (using a so-called soft or hard thresholding), and to transform the data back into the original domain. Such non-linear thresholding estimator can be computed in any orthogonal basis (as Fourier or cosine), and Donoho and Johnstone have proved that its performance is close to an ideal coefficient selection and attenuation [1]. And since the efficiency of the estimator depends on the rate of decay of the sorted decomposition coefficients, it is well known that the choice of Wavelet series outperforms the choice of Fourier or cosine series in the representation of piecewise-smooth signals (see e.g.[2, $3])$ : thanks to the wavelets time-localization, the decay of wavelet coefficients in the neighborhood of discontinuities is faster that the decay of Fourier coefficients. However, wavelet thresholding method is still a regularization process and the estimator presents oscillations in the vicinity of signal's discontinuities. Such oscillations are very close to the Gibbs phenomena exhibited by Fourier thresholding, although they are more local and of smaller amplitude. They are called pseudo-Gibbs phenomena.

To partially reduce these artifacts, a workaround has been proposed by Coifman and Donoho [4] : their translation invariant thresholding algorithm consists in applying the thresholding process to translated versions of the original signal, and in averaging them. It comes down to apply the classical threshold process on a frame wavelet expansion, the dyadic wavelets [5], instead of an orthogonal wavelet basis. If the resulting procedure, called SpinCycle by Coifman and Donoho, really reduces the pseudo-Gibbs phenomena compared to the original VisuShrink procedure, they are still highly visible. The method we are presenting in this paper performs a far better artifact reduction, so that in most cases the pseudo-Gibbs phenomena simply vanish. Let us mention another approach of artifact free wavelet denoising recently introduced by Dragotti and Vetterli in [6]. These authors propose to apply a vector thresholding in place of the classical scalar thresholding, and to replace remaining vectors by the closest footprint obtained as the significant wavelet coefficients generated by singularities of piecewise polynomial functions. In this way, they ensure a denoising free of pseudo-Gibbs phenomena. However the computation and the recording of all footprints are pretty heavy, and the extension of this method to signals of higher dimensions, such as images, a challenging program. The method we are introducing keeps a low complexity and can be easily extended to image denoising. 
Since classical wavelet thresholding does not introduce artifacts in regular parts of a signal, we focus our attention on denoising piecewise smooth signals with sharp discontinuities. The method we are introducing is working in two steps :

- Apply a traditional wavelet denoising method, such as a hard thresholding, and record the location of the remaining coefficients in a map $M$. Donoho recommends to use the soft thresholding VisuShrink instead of the hard one in order to reduce the artifacts [7]. Although the algorithm we are presented can be used with VisuShrink, it is mathematically relevant with a hard thresholding only.

- Restore the denoised signal using a total variation minimization approach, subject to the constraint that the restored signal has the same remaining wavelet coefficients than the denoised one. This step removes the pseudo-Gibbs phenomena and restores sharp discontinuities, while the other structures are preserved.

The idea of using a total variation minimization method to restore a noisy signal has been introduced for the first time by Rudin, Osher and Fatemi in [8], in the context of image denoising. They propose to minimize the total variation of the signal subject to a fidelity constraint, so that the restored signal has a lower total variation while it remains close to the original one. The noise is reduced while discontinuities are preserved, in contrast with classical regularization techniques where discontinuities are smoothed. Following this work, several constraints and functionals to minimize have been proposed (see e.g. $[9,10]$ ).

We do not propose to use the total variation in order to remove the noise : the first step of our method, the wavelet denoising, performs this task very well and even better than the total variation approach. We propose to reconstruct a signal with minimal total variation such that its wavelet coefficients are the same than the remaining wavelet coefficients of the denoising signal. This main idea results simply in the following remark : if step one is correctly performed, the unknown original noiseless signal has the same wavelet coefficients than the denoised one in the location given by the map $M$. Traditional wavelet denoising algorithm makes the choice of setting the coefficients outside $M$ to 0 , leading oscillations in the vicinity of discontinuities. Because of the strong dependency between wavelet coefficients in the original noiseless signal, this is far from an optimal choice. We propose to set the coefficients outside $M$ to the values that minimize the total variation of the reconstructed signal, so that occurence of oscillations is discouraged.

The following details steps one and two of our method. Then, we present the algorithm used to minimize the total variation. We end with some experimental results which show that our approach allows, in addition to the artifact removal, a great SNR enhancement. Let us mention that the theory developed here is valid with any orthogonal bases, thought we are presenting it in the framework of wavelet bases.

\section{WAVELET DENOISING}

Let $\left(\psi_{j, k}\right)_{j, k \in K}$ be an orthogonal basis of wavelets on the interval $I=[a, b]$ as described by Cohen, Daubechies and Vial [11], so that we can write any signal $u \in L^{2}(I)$ as the sum of the serie

$$
u=\sum_{j, k \in K}<u, \psi_{j, k}>\psi_{j, k}
$$

where

$$
<u, \psi_{j, k}>=\int_{I} u(x) \psi_{j, k}(x) d x .
$$

Let us define the hard thresholding operator $\tau$ to be

$$
\tau(x)= \begin{cases}x & \text { if }|x| \geq \lambda, \\ 0 & \text { if }|x|<\lambda .\end{cases}
$$

In the case of soft thresholding, the operator $\tau$ is

$$
\tau(x)= \begin{cases}x-\operatorname{sgn}(x) \lambda & \text { if }|x| \geq \lambda, \\ 0 & \text { if }|x|<\lambda .\end{cases}
$$

The denoised signal using wavelet thresholding is simply

$$
u_{0}=\sum_{j, k \in K} \tau\left(<u, \psi_{j, k}>\right) \psi_{j, k} .
$$

The map $M$ is defined by

$$
M=\left\{(j, k) \in K:\left|<u, \psi_{j, k}>\right| \geq \lambda\right\} .
$$

If the noisy signal can be written $u=\tilde{u}+w$, with $\tilde{u}$ the noiseless signal to estimate and $w$ an additive Gaussian white noise of standard deviation $\sigma$, the threshold $\lambda$ is often set to $\sigma \sqrt{2 \log N}, N$ being the number of samples of the digital signal : in that case the estimator is the best in the min-max sense as $N$ tends to infinity [1].

\section{CONSTRAINED TOTAL VARIATION MINIMIZATION}

The total variation of any unidimensional signal $v$ in $I$ is

$$
J(v)=\sup _{\left(x_{l}\right)} \sum_{l=1}^{L}\left|v\left(x_{l}\right)-v\left(x_{l-1}\right)\right|
$$

where the supremum is on all sequences $\left(x_{l}\right)$ such that $a \leq$ $x_{1}<x_{2}<\ldots<x_{L} \leq b$. Let $U$ be the constraint space

$$
U=\left\{v: \forall(j, k) \in M,<v, \psi_{j, k}>=<u, \psi_{j, k}>\right\} .
$$


This is an affine space with direction given by the linear space

$$
U_{0}=\left\{v: \forall(j, k) \in M,<v, \psi_{j, k}>=0\right\} .
$$

We have to solve the variational problem

$$
\text { find } u^{*} \in U \text { such that } J\left(u^{*}\right)=\inf _{v \in U} J(v) .
$$

Since $J$ is a convex function and $U$ a convex set, any solution $u^{*}$ of (10) is given by

$$
\forall t>0, u^{*}=P\left(u^{*}-t \frac{\partial J}{\partial v}\left(u^{*}\right)\right),
$$

for $P$ the affine projector onto $U$ that minimizes the distance.

\section{ALGORITHM}

The algorithm is a straightforward discrete approximation of the previous equations. Wavelet denoising is a simple process which involves a FWT (Fast Wavelet Transform) followed by thresholding and by an inverse FWT.

We numerically solve the variational problem using a well-known gradient descent algorithm combining a projection on the constraint :

$$
u_{k+1}=P\left(u_{k}-t_{k} \frac{\partial J}{\partial v}\left(u_{k}\right)\right),
$$

where $u_{0} \in U$ is the denoised signal by wavelet thresholding and $t_{k}>0$ is the step chosen in order to obtain the convergence. Let $P_{0}$ be the orthogonal projection onto $U_{0}$. Since we have

$$
P(v)=u+P_{0}(v-u),
$$

we can write

$$
u_{k+1}=u_{k}-t_{k} P_{0}\left(\frac{\partial J}{\partial v}\left(u_{k}\right)\right)
$$

and this equation can be easily computed : the signal $\frac{\partial J}{\partial v}\left(u_{k}\right)$ is projected onto $U_{0}$ using the FWT, followed by the cancellation of coefficients belonging to $M$ and by an inverse FWT.

The overall complexity of the algorithm remains of the same order than the FWT, that is $O(N)$, which is lower than the SpinCycle complexity $(O(N \log N))$. However, because of the fixed number of iterations needed by the gradient descent, the effective computational time is greater than the one associated to SpinCycle, at least for signals of reasonable size.

\section{EXPERIMENTAL RESULTS}

We have implemented the algorithm using the tools given by the free MegaWave2 software [12].

We present two experiments. The first one consists in denoising a synthetic signal containing a sharp discontinuity (a step) and two discontinuities of second order (a ramp). A Gaussian white noise has been added following the model $u=\tilde{u}+w$. Figure 1 displays the signal $\tilde{u}$, Figure 2 the signal $u$, Figure 3 the wavelet-denoised signal $u_{0}$ and Figure 4 the restored signal $u_{k}$ for $k=10000$. The estimator $u_{k}$ is far better than $u_{0}$, either in terms of SNR or visually. Fair results are still obtained with much lower $k$ (as low as $k \simeq 10$ ). The second experiment is obtained from a natural noisy signal $u$, which follows our assumption of a piecewise smooth noiseless signal $\tilde{u}$. The signal $u$ in Figure 5 corresponds to a line of a digital image, which is a snapshot of an office. Figure 6 shows the signal $u_{0}$ and Figure 7 the signal $u_{k}$. Once again, the visual aspect of $u_{k}$ is far better than $u_{0}$.

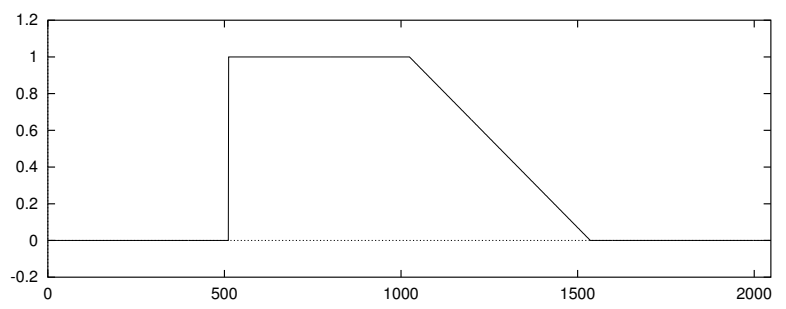

Fig. 1. Original step-ramp function $\tilde{u}$.

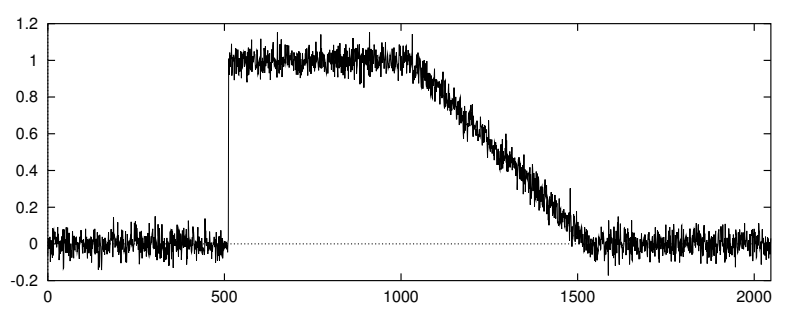

Fig. 2. Noised step-ramp function $u$, obtained by adding to $\tilde{u}$ a Gaussian white noise of $\sigma=0.05$. SNR $=18.9 \mathrm{db}$.

\section{CONCLUSION}

We have presented a general framework to perform artifactfree denoising with wavelets. The method was explained and illustrated in the case of unidimensional signals, but it can be easily extended to signals of higher dimensions, and in particular to images for which the piecewise-smooth assumption is highly relevant. A slight modification of the constraint may also be performed in order to achieve restoration of signals and images that have been compressed within an orthogonal basis. 


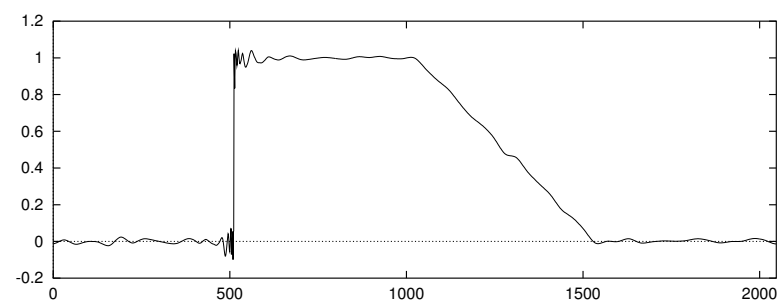

Fig. 3. Denoised step-ramp function $u_{0}$, obtained by wavelet hard thresholding. SNR=31.0 db. Normalized Total Variation NTV $=0.0023$.

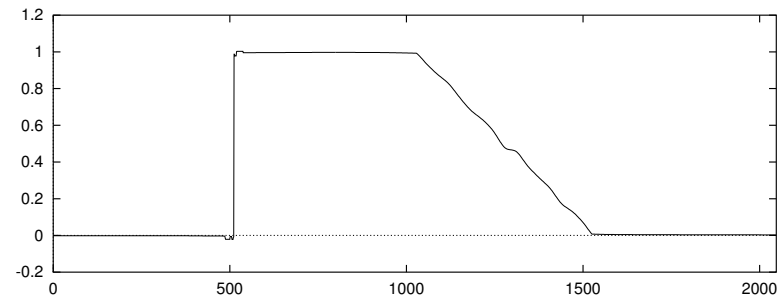

Fig. 4. Denoised step-ramp function $u_{k}$, obtained by our method. $\mathrm{SNR}=37.8 \mathrm{db}$. NTV=0.0010.

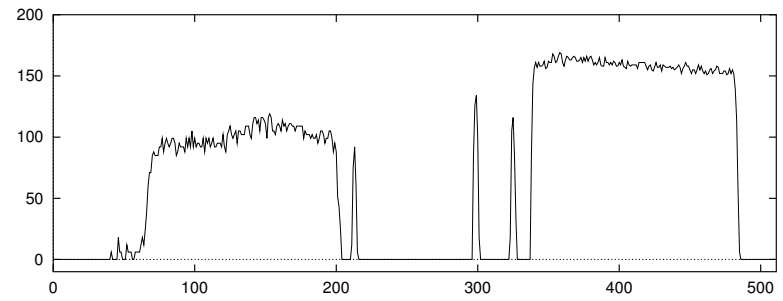

Fig. 5. Real signal $u$ extracted from a line of a digital image (view of an office).

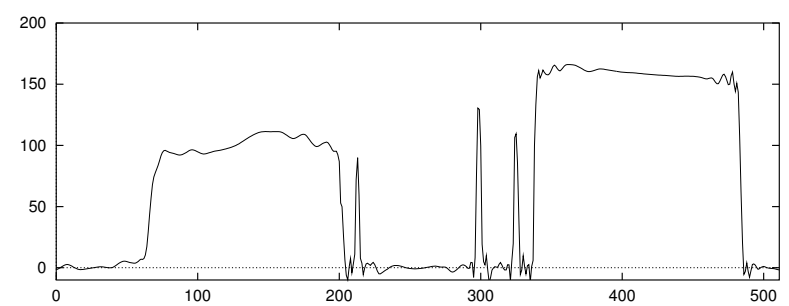

Fig. 6. Denoised line of the image, obtained by wavelet hard thresholding. NTV=3.244.

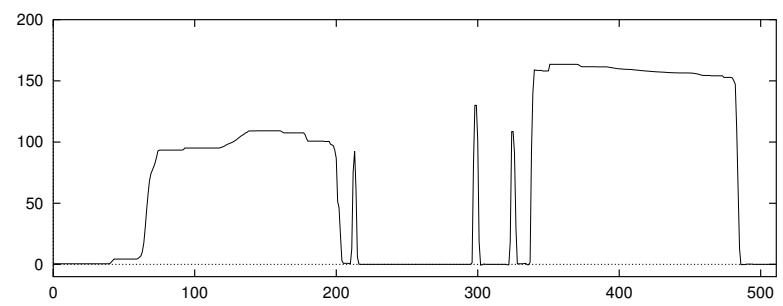

Fig. 7. Denoised line of the image, obtained by our method. $\mathrm{NTV}=2.365$.

\section{Acknowledgments}

The authors are grateful to François Malgouyres, Lionel Moisan and Bernard Rougé for useful discussions.

\section{REFERENCES}

[1] D. Donoho and I. Johnstone, "Ideal spatial adaptation via wavelet shrinkage," Biometrika, vol. 81, pp. 425455, 1994.

[2] A. Cohen, J. Froment, and J. Istas, "Analyse multirésolution des signaux aléatoires," Note aux C.R. Acad. Sci. Paris, vol. t.312, no. I, pp. 567-570, 1991, Analyse mathématique.

[3] A. Cohen and J.P. D'Alès, "Nonlinear approximation of random functions," SIAM J. Appl. Math., vol. 57, no. 2, pp. 518-540, 1997.

[4] R.R. Coifman and D.L. Donoho, "Translationinvariant de-noising," in Wavelets and Statistics, A. Antoniadis and G. Oppenheim, Eds., pp. 125-150. Springer-Verlag, 1995.

[5] S. Mallat and S. Zhong, "Characterization of signals from multiscale edges," IEEE Trans. Pattern Recog. and Machine Intell., vol. 14, no. 7, pp. 710-732, 1992.

[6] P.L. Dragotti and M. Vetterli, "Shift-invariant gibbs free denoising algorithm based on wavelet tranform footprints," in Proc. of SPIE'2000, Wavelet Application in Signal and Image Processing, 2000.

[7] D. Donoho, "De-noising by soft-thresholding," IEEE Trans. on Info. Theory, vol. 41, pp. 613-627, 1995.

[8] L.I. Rudin and E. Fatemi S. Osher, "Nonlinear total variation based noise removal algorithms," Physica $D$, vol. 60, pp. 259-268, 1992.

[9] A. Chambolle and P-L. Lions, "Image recovery via total variation minimization and related problems," $\mathrm{Nu}$ merische Mathematik, vol. 76, pp. 167-188, 1997.

[10] S. Durand, F. Malgouyres, and B. Rougé, "Image deblurring, spectrum interpolation and application to satellite imaging," ESAIM:COCV Control, Opt. and Cal. of Var, vol. 5, pp. 445-475, 2000.

[11] A. Cohen, I. Daubechies, and P. Vial, "Wavelets and fast wavelet transforms on an interval," Applied and Comput. Harmonic Ana., vol. 1, pp. 54-81, 1993.

[12] J. Froment and L. Moisan (Eds), "Megawave2," a free and open-source Unix image processing software for reproducible research, available at http://www. cmla.ens-cachan.fr. 